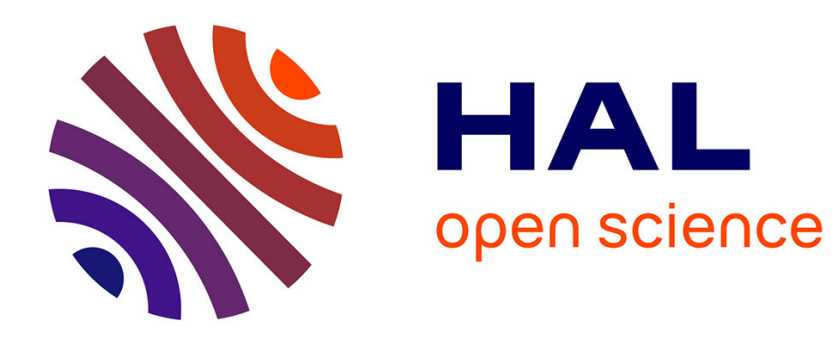

\title{
Bifurcation theory and localization phenomena
}

Jean Sulem

\section{To cite this version:}

Jean Sulem. Bifurcation theory and localization phenomena. European Journal of Environmental and Civil Engineering, 2010, 14 (8-9), pp.989-1009. hal-00540003

\section{HAL Id: hal-00540003 https://hal.science/hal-00540003}

Submitted on 25 Nov 2010

HAL is a multi-disciplinary open access archive for the deposit and dissemination of scientific research documents, whether they are published or not. The documents may come from teaching and research institutions in France or abroad, or from public or private research centers.
L'archive ouverte pluridisciplinaire HAL, est destinée au dépôt et à la diffusion de documents scientifiques de niveau recherche, publiés ou non, émanant des établissements d'enseignement et de recherche français ou étrangers, des laboratoires publics ou privés. 


\title{
Bifurcation theory and localization phenomena
}

\author{
Jean Sulem \\ UR Navier-CERMES \\ Ecole des Ponts Paris Tech / Université Paris-Est \\ 6-8 Av. Blaise Pascal \\ Cité Descartes Champs sur Marne \\ 77455 Marne la Vallée Cedex 2 France \\ sulem@cermes.enpc.fr
}

ABSTRACT. The principles of strain localization analysis as applied to geomaterials are presented. Emphasis is given to the effects of fluid and temperature in the occurrence and development of instabilities.

RÉSUMÉ. Dans cet article, on présente les principes de l'analyse de localisation des déformations dans les géomatériaux. En particulier, on met l'accent sur les effets du fluide et de la température sur l'apparition et le développement des instabilités.

KEYWORDS: strain localization, bifurcation, constitutive models, fluid, temperature.

MOTS-CLÉS: localisation des déformations, bifurcation, loi de comportement, fluide, température.

Revue. Volume $\mathrm{X}-\mathrm{n}^{\circ} \mathrm{x} /$ année, pages 1 à $\mathrm{X}$ 


\section{Introduction}

Failure of many engineering structures is characterized by the formation and propagation of a failure plane. Laboratory experiments as well as in field observations that the orientation of the failure plane (or fault surface) is controlled by the directions of the principal stresses. In the well established Mohr-Coulomb's theory, the inclination $\theta$ of the failure surface with respect to the direction of the minimum (in absolute value) principal stress is given as where $\phi$ is the friction angle of the material. For typical values of Coulomb friction angle $\phi, 30^{\circ}$ to $50^{\circ}$, values of $\theta$ range from $60^{\circ}$ to $70^{\circ}$, which is comparable with the range of observed failure plane inclinations. Mohr-Coulomb's theory is commonly used in geomechanics as the dominant feature in the behaviour of geomaterials behaviour is its frictional character. The orientation of a failure surface can be deduced from the knowledge of the orientation of the principal stresses (not their magnitude) and of one material property (the internal friction angle). Inversely, the orientation of the principal stresses can be simply deduced, using Mohr-Coulomb's theory, from the orientation of the failure plane and the knowledge of the friction coefficient of the material.

Although the simplicity of this approach has made it very useful, the predictions of Mohr-Coulomb's theory have been criticized because it is commonly observed that the complete inelastic response of a material influences the conditions of incipient failure and not only one material parameter such as the internal friction angle.

Moreover for studying the response of a structure in the post-failure regime when the strength is suddenly dropping, one has to understand what failure physically means. This is exactly the question addressed in the early seventies by Professor Ioannis Vardoulakis and for which he produced his pioneering work and milestone contributions. The first observation was that what appears as a failure plane is in fact a zone of localized shear deformation. Thus the study and the modelling of strain localisation phenomena has proven to be very useful in the understanding of failure mechanisms. Based on the theoretical studies of material stability as developed by Hadamard (1903) for elastic materials and later extended by Thomas (1961), Hill (1962) and Mandel (1966) for inelastic materials, the localisation process is seen as an instability that can be predicted from the pre-failure constitutive behaviour of the material. The conditions for the onset of localisation are thus established by seeking the possible critical conditions for which the constitutive equations of the material (in the pre-localised stage) may allow the existence of a bifurcation point for which the deformation mode will localize into a planar band (Rice 1976, Vardoulakis, 1976). In this approach, the initiation of failure in the form of the incipient of a shear band is modelled as a constitutive instability and consequently a great number of studies have been dealing with the development of appropriate constitutive relationships which can predict satisfactorily the onset of shear banding. Geomaterials are characterised by a non-associated and non-coaxial plastic behaviour and this has important effects on the localisation process (Rudnicki \& 
Rice, 1975). Numerous experimental studies on soils and rocks have been performed to understand the physical processes which control strain localization and validate the theoretical and constitutive concepts (e.g. Vardoulakis, 1980, Sulem et al. 1999, Bésuelle et al., 2000, Desrues \& Viggiani, 2004).

Shear band formation in the form of localized shear deformation is not the only possible localized deformation mode. Zones of localized deformation are sometimes observed in the direction normal to the maximum compressive stress as observed in highly porous rocks. These structures are interpreted as compactions bands.

The problem of modelling localized deformation in geomaterials is quite a challenging task, due to the difficulties which are encountered while dealing with softening materials and moving elastoplastic boundaries. It is however one of the most interesting bifurcation problems: Asking the question of possible spontaneous change of the deformation mode for a given loading history and subsequent evolution of this secondary deformation mode, one may search for the conditions of uniqueness and stability of the corresponding boundary value problem. Thus as first presented by Mandel $(1964,1966)$ questions of uniqueness and stability of solutions arise naturally within the context of shear-band analysis. It turns out that the result of such analyses dealing with geomaterials depends primarily on the assumed physical non-linearities which are inherent to the underlying constitutive description and is in a lesser degree influenced by geometrical non-linearities.

It is not possible in a single paper to review the important literature published in the last forty years on the subject. We will thus choose to address some points and give a flavour of the mathematical and physical background of bifurcation analysis as applied to geomechanics with some special emphasis on the effects of fluid and temperature.

\section{Notions of bifurcation and loss of stability}

As the words bifurcation and instability are frequently used in the discussions about failure of geomaterials, it is important to clarify the meaning of these terms. The reader can find in Chambon et al (2004) a very clear and solid presentation of these notions and also guidelines when dealing with them. In this paper presented in a previous ALERT school, these authors have strongly emphasized the importance of the use of an appropriate vocabulary in order to avoid misunderstandings and ambiguous statements.

The word stability refers to Lyapunov (1892) theoretical results and makes reference with the evolution in time of a well defined perturbation on a given mechanical system. Physically, it means that a system is stable (in the sense of Lyapunov) if a little disturbance of the initial conditions will not increase with time. The word bifurcation refers to the loss of uniqueness of the solutions of a given boundary problem. These notions of stability and bifurcation thus refer to a 
mechanical system with well defined boundary and initial conditions. For engineering applications, stability and bifurcation analyses usually involve a finite domain subjected to non homogeneous stress and strain states. Interesting results can also be obtained when the studied system is restricted to a material element which is a representative volume of material in a homogeneous state of stress and strain. Considering the evolution of this element, we ask the question of possible alternative deformation modes for the next loading increment. Spontaneous change of deformation for example in the form of localized deformation in a planar band corresponds thus to a bifurcation state (loss of uniqueness) and corresponds to a material instability. Shear localization can be preceded with diffuse non homogeneous deformation modes such as bulging or barrelling especially in axisymmetric compression tests (Sulem \& Vardoulakis, 1990, Chau \& Rudnicki, 1990). These instabilities are of material and geometric nature. A discussion on localized and diffuse bifurcation modes can be found in the recent paper of Nicot \& Darve (2010) in relation with the non associate character of the constitutive law. The role of the loading control parameters on the occurrence of homogeneous or non homogeneous bifurcations has been investigated by Nova (2004) with the concept of controllability in geotechnical testing.

\section{Shear band model}

Extensive presentation of shear band analysis in geomaterials can be found in Vardoulakis and Sulem (1995) (see also Desrues \& Chambon 2002, Bésuelle \& Rudnicki, 2004 for a review of recent studies). The strain localisation analysis consists in searching the incipient of a shear band in a solid as a mathematical bifurcation condition for the deformation field. Considering an infinitesimal neighbourhood of a point in an elastic-plastic solid which is homogeneous as for the constitutive law and stress state, the strain localisation phenomenon is understood as the appearance of a discontinuity in strain rates which marks the onset of nonuniform response. Such a bifurcation of the velocity gradient along a loading path can be caused by material destabilising effects such as softening and lack of plastic normality in the constitutive law, as well as geometrical destabilising effects such as large deformation affecting equilibrium equations. This bifurcation condition is obtained from (a) the constitutive relationships of the material, (b) the conditions of mechanical equilibrium across the shear band boundary and (c) the kinematic compatibility conditions which expresses that the velocity field is to be continuous (no material discontinuity). The latter condition implies that only the normal component of the velocity gradient across the shear band is discontinuous whereas the tangential one is continuous (weak discontinuity). The above conditions describe the so-called 'continuous' bifurcation modes. It has been shown that the critical state for continuous bifurcation precedes the one for 'discontinuous' bifurcation where a discontinuity of the velocity field itself (and not only its gradient) is considered (Simo et al. 1993). Non-trivial solution for the condition of continuous bifurcation is 
a necessary condition for the shear band existence and provides both the shear band orientation and the deformation jump across the shear band. Rudnicki and Rice (1975) and Rice (1976) have obtained solutions for realistic elasto-plastic constitutive relationships for geomaterials.

\section{Strain localization criterion}

According to the definition proposed by Hill (1962), a shear band is viewed as a thin layer that is bounded by two parallel material discontinuity surfaces of the incremental displacement gradient (Figure 1). These material discontinuity surfaces $\mathrm{D}^{(1)}$ and $\mathrm{D}^{(2)}$ are called shear-band boundaries and their distance, $2 \mathrm{~d}_{\mathrm{B}}$, is the thickness of the shear-band. Within the frame of constitutive theories without material length, the shear-band thickness $d_{B}$ is undetermined.

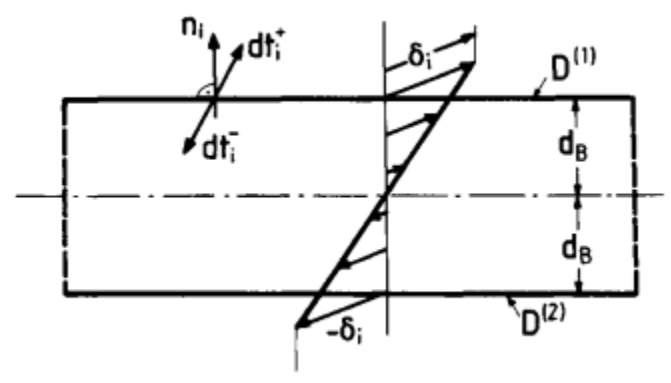

Figure 1. Model of a deforming shear-band with heat and fluid fluxes

Let denote by $\boldsymbol{n}$ the unit vector normal to the band. Inside the band the incremental displacement field $\Delta \boldsymbol{u}^{1}$ depends only on the position across the band; outside the band the rate of deformation is assumed to remain homogeneous. Assuming that the displacement field is continuous across the band, then according to Maxwell theorem, only the normal derivative of this field may be discontinuous across the band. Accordingly the following kinematic compatibility conditions hold:

$$
\left[\Delta u_{i}\right]=0 \text { and }\left[\partial_{j} \Delta u_{i}\right]=g_{i} n_{j}
$$

where [.] denotes the jump of the quantity across the shear band boundary. Note that as emphasized by Bésuelle and Rudnicki (2004), the form of equation [1] requires that $\left[\partial_{j} \Delta u_{i}\right]$ has a vanishing intermediate eigenvalue and thus contains a plane of zero incremental displacement. Consequently, localization is favoured when the prebifurcation, homogeneous field contains a plane of zero extension rates, as in plane strain whereas highly destabilizing effects as strong softening behaviour is needed to generate shear band formation in axisymmetric deformation. 
Incremental strains and stresses are linked through the constitutive relationships:

$$
\Delta \sigma_{i j}=C_{i j k l} \partial_{l} \Delta u_{k}
$$

Equilibrium across the shear band boundary implies the following static compatibility condition:

$$
\left[\Delta \sigma_{i j}\right] n_{j}=0
$$

We observe that there are two possibilities, namely that the constitutive behaviour across the shear band boundaries is either continuous or discontinuous. Concerning discontinuous bifurcations one has to examine the possibility that elastic unloading occurs outside the shear band while continued elastic-plastic loading occurs within the band. If the elasto-plastic constitutive law admits a single smooth yield surface and plastic potential, Rice and Rudnicki (1980) have shown that continuous bifurcation analyses provide the lower limit to the range of deformations for which discontinuous bifurcations can occur. Accordingly, we restrict ourselves here to the first possibility of continuous constitutive behaviour, namely $\left[C_{i j k l}\right]=0$.

Using the constitutive relationships [2], the static compatibility condition [3] and the kinematic compatibility conditions [1], we finally obtain

$$
\Gamma_{i k} g_{k}=0
$$

where $\Gamma_{i k}=C_{i j k l} n_{j} n_{l}$ is the acoustic tensor. It follows that weak stationary discontinuities for the incremental displacement exist only if the acoustic tensor is singular:

$$
\operatorname{det} \Gamma=0
$$

Equation [5] is the characteristic equation in terms of the direction cosines $n_{\mathrm{i}}$ of a statically, kinematically and materially admissible discontinuity surface. If the characteristic equation provides real solutions for the direction cosines $n_{\mathrm{i}}$, discontinuity surfaces for the incremental displacement gradient exist and may also develop in due course of the deformation. Equation [5] is thus the localization criterion.

\section{Strain localization, loss of ellipticity and vanishing speed of acceleration waves}

The governing equations of an incremental boundary problem are obtained from the equations of equilibrium $\partial_{j} \Delta \sigma_{i j}=0$ (for simplicity we omit here the body forces), the constitutive relationships of the form given by equation [2], and the boundary conditions (prescribed tractions or displacements at the boundary of the considered body). Incorporating the constitutive equations [2] into the equilibrium 
equations and assuming piecewise linear incremental constitutive equations yields the following second order differential system:

$$
C_{i j k l} \partial_{j} \partial_{l} \Delta u_{l}=0
$$

The ellipticity condition of the above differential system is expressed as follows:

$$
\forall \mathbf{n}, C_{i j k l} n_{j} n_{l} \text { is strictly definite positive. }
$$

Consequently, the localization criterion [5] corresponds to the state of loss of ellipticity of the governing equations. They change type and from elliptic they turn to hyperbolic. Shear bands are thus identified with the characteristic lines of the governing hyperbolic partial differential equations.

Let us consider now the propagation of acceleration waves in a solid body along the direction $\mathbf{n}$. Acceleration waves are weak discontinuities of the various mechanical fields across wave-fronts which propagate with the speed $c$. One can show that the propagation speed $c$ is the solution of an eigen-value problem (cf. Vardoulakis \& Sulem 1995) and that $c$ is obtained from the following equation:

$$
\operatorname{det}\left(\Gamma_{i k}-\rho c^{2} \delta_{i k}\right)
$$

where $\rho$ is the density of the material. Thus, if the acoustic tensor is strictly definite positive, all the velocities of acceleration waves are real. When all waves are able to propagate with real velocity, the material is stable in a dynamic sense. This is called the Hadamard's stability criterion (Hadamard, 1903) first established for hyperelastic materials. Consequently the localization criterion [5] corresponds to a state for which the velocity of wave propagation in the direction normal to the band is null (stationary wave).

\section{Shear band formation in element tests on rocks}

\section{Drucker-Prager model}

As seen above, the localization criterion depends on the constitutive relation. The Drucker-Prager plasticity model with non associate flow rule is commonly used for porous rocks. The yield surface and the plastic potential are expressed as

$$
F=\bar{\tau}-\mu(q-\sigma) ; Q=\bar{\tau}+\beta \sigma
$$

where $\sigma=\sigma_{k k} / 3$ is the mean stress (negative in compression), $\bar{\tau}=\sqrt{s_{i j} s_{i j} / 2}$ is the Mises equivalent stress (with $s_{i j}=\sigma_{i j}-\sigma \delta_{i j}$ ), $\mu$ is the friction coefficient and $\beta$ is the dilatancy coefficient. For low-porosity rock, inelastic response is dilatant and $\beta>0$; however, compressed high-porosity rock typically experiences initial 
compaction, followed by either dilation or further compaction, depending upon the stress state. Negative values for the dilatancy coefficient and negative values for the friction coefficient at high mean stress can be thus observed as for cap yield surface. The critical value of the hardening modulus for which the localization is satisfied (equation [5]) is given by (Rudnicki \& Rice, 1975):

$$
\frac{H_{c}}{G}=\frac{1+v}{9(1-v)}(\beta-\mu)^{2}-\frac{1+v}{2}\left(N+\frac{\beta+\mu}{3}\right)^{2}
$$

where $G$ is the elastic shear modulus, $v$ is the Poisson's ratio, $\mu$ is the friction coefficient and $\beta$ is the dilatancy coefficient $N$ is the normalized intermediate principal deviatoric stress $\left(N_{i j}=s_{i j} / \bar{\tau}\right)$ and varies from $-1 / \sqrt{3}$ for axisymmetric extension $\left(\sigma_{1}=\sigma_{2}>\sigma_{3}\right)$ to $1 / \sqrt{3}$ for axisymmetric compression $\left(\sigma_{1}>\sigma_{2}=\sigma_{3}\right)$. The value $N=0$ corresponds to pure shear $\left(\sigma_{1}=-\sigma_{3}, \sigma_{2}=0\right)$. The shear band is in a plane parallel to the intermediate principal stress and its normal is inclined with an angle $\theta_{B}$ with respect to the $\sigma_{3}$ - direction (most compressive direction) expressed as (Rudnicki \& Olsson, 1998)

$$
\theta_{B}=\frac{\pi}{4}+\frac{1}{2} \arcsin \alpha, \text { with } \alpha=\frac{(2 / 3)(1+v)(\beta+\mu)-N(1-2 v)}{\sqrt{4-3 N^{2}}}
$$

The above equations give the largest critical value of the hardening modulus and the shear band orientation as established by Rudnicki and Rice (1975). As mentioned by Perrin and Leblond (1993), these solutions are valid only if $|\alpha| \leq 1$, thus when the magnitude of $\mu+\beta$ is small enough

$$
\frac{N(1-2 v)-\sqrt{4-3 N^{2}}}{2(1+v) / 3} \leq \beta+\mu \leq \frac{N(1-2 v)+\sqrt{4-3 N^{2}}}{2(1+v) / 3}
$$

The case for which $\beta+\mu>\frac{N(1-2 v)+\sqrt{4-3 N^{2}}}{2(1+v) / 3}$ corresponds to the formation of dilation bands in the direction normal to the least compressive principal stress, $\theta_{B}=\pi / 2$, whereas, the case for which $\beta+\mu<\frac{N(1-2 v)+\sqrt{4-3 N^{2}}}{2(1+v) / 3}$ corresponds to the formation of compaction bands in the direction parallel to the least compressive principal stress, $\theta_{B}=0$ (Issen \& Rudnicki, 2000). The corresponding critical hardening modulus has the form

$$
\frac{H_{c}^{k}}{G}=\frac{1+v}{9(1-v)}(\beta-\mu)^{2}-\frac{1+v}{1-v}\left(\frac{1}{2} N_{k}-\frac{\beta+\mu}{3}\right)^{2}-\left(1-\frac{3}{4} N_{k}^{2}\right)
$$


where $k=1$ for the dilation bands and $k=3$ for the compaction bands and $N_{1}$ and $N_{3}$ are the least and most compressive principal values of the normalized deviatoric stress tensor. These solutions for the critical hardening modulus and the orientation of the band are continuous over the limits of equation [12].

For plane strain deformation an approximate solution for the shear band inclination (equation [11]) has been given by Vardoulakis (1980)

$$
\theta_{B}=\frac{\pi}{4}+\frac{\phi_{p}}{4}+\frac{\psi_{p}}{4}
$$

where $\phi_{p}$ and $\psi_{p}$ are respectively the friction angle and the dilatancy angle at peak values. This expression was first proposed by Arthur et al. (1977) on the basis of experimental observations.

\section{Non coaxial plasticity}

In classical flow theory of plasticity, the direction of the plastic deformation is fixed with respect to the normal to the plastic potential. Consequently, the direction is fixed by the current state of stress and does not depend upon the direction of the stress increment. The plastic deformation rate possesses the same principal axes as the stress tensor, which means that it is coaxial to the stress tensor. This is a strong assumption and its consequences on the prediction of shear localization have been discussed in many papers (e.g. Rudnicki \& Rice, 1975, Vardoulakis, 1980, Papamichos \& Vardoulakis, 1995). In order to arrive to better predictions for shearband formation, one has to abandon the concept of classical coaxial plasticity flow rule and resort to hypoplasticity flow rules, which consider one way or the other the effect of stress rate. Rudnicki \& Rice (1975) have considered the contribution of a non-coaxial term motivated by a yield vertex plasticity model. Other examples of non-coaxial constitutive models have been proposed in the form of a deformation theory of plasticity (e.g. Vermeer and Schotman, 1986, Sulem \& Vardoulakis, 1990). More generally, the incrementally non-linear laws of Darve, 1985, Chambon and Desrues, 1989 developed for granular soils and rocks are non-coaxial.

These modifications of the plasticity flow rule result in significant changes in material response for non-proportional loading paths (Desrues \& Chambon, 2002, Bésuelle \& Rudnicki, 2004).

\section{Cataclastic shear banding}

At high confinement, suppressed dilatancy may lead to grain crushing or cataclasis inside the shear band (El Bied et al. 2002) as shown in Figure 2 which in turn leads to substantial porosity and permeability reduction (Sulem \& Ouffroukh, 2006). At large scale, similar phenomena are observed in faulted zones when sheared. Usually in fault zones, two main domains can be identified: a fault core of small thickness constituted of highly comminuted ultra-cataclasites is surrounded with a damage zone which consists of fractured host rock (e.g Chester \& Chester, 1998). The 
ultracataclastic structure is the results of numerous earthquake ruptures. As shown for example in a recent study of Sulem et al (2004) dedicated to the characterisation of Aigion fault material in the Gulf of Corinth (Greece), the permeability of the fault core is very low so that this zone acts as an impervious barrier to transverse fluid flow, whereas the highly fractured damage zone around acts mostly as conduits for nearly along-strike flow. Similar observations can be found in the work of Wibberley and Shimamoto (2005).

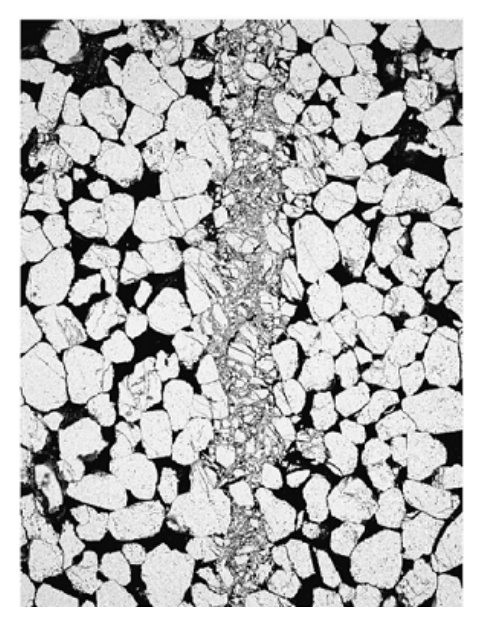

Figure 2. Cataclastic shear banding in Fontainebleau sandstone (El Bied et al., 2002).

\section{Post-bifurcation behavior}

The various drawbacks and shortcomings of the classical continuum theory in connection with strain localization are related to the fact that in the post-bifurcation regime we deal in general with mathematically ill-posed governing equations. The origin of this undesirable situation can be traced back to the fact that conventional constitutive models do not contain material parameters with dimension of length, so that the shear band thickness (i.e. the extent of the plastically softening region) is undetermined. We can say that localization of deformation leads to a change in scale of the problem so that phenomena occurring at the scale of the grain cannot be ignored anymore in the modelling of the macroscopic behavior of the material. Then it appears necessary to resort to continuum models with microstructure to describe correctly localization phenomena. These generalized continua usually contain additional kinematical degrees of freedom (Cosserat continuum) and/or higher deformation gradients (higher grade continuum). Cosserat continua and higher grade continua belong to a general class of constitutive models which account for the materials micro-structure. The contemporary formulation of these models are based 
on the work of Mindlin (1964b), Germain (1973a,b). Rotation gradients and higher velocity gradients introduce a material length scale into the problem, which as already mentioned is necessary for the correct modeling of localization phenomena. This idea was widely publicized by the paper of Mühlhaus and Vardoulakis (1987). In this case the underlying mathematical problem describing localization phenomena is 'regularized' and the governing equations remain elliptic. Moreover, this technique allows robust computations to follow the evolution of the considered system in the post-bifurcation regime and to extract additional information such as the shear band thickness or to assess the effect of scale. In the two last decades, large scale numerical simulations which account for higher order continuum effects have been developed (Papanastasiou \& Vardoulakis, 2002, Zervos et al. 2001, Matsushima et al. 2002, Sieffert et al. 2009).

\section{Effects of pore fluid and temperature}

The mechanical interaction of pore fluid and deformation affects the occurrence and evolution of localization. Moreover, thermal weakening mechanisms as for example by thermal pressurization of the pore fluid induced by frictional heating have destabilizing effects (e.g. Rice, 2006, Sulem et al. 2007, Sulem, 2009).

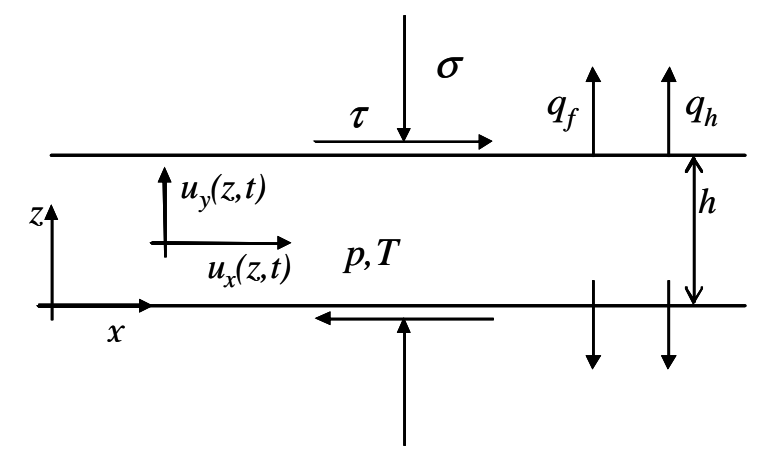

Figure 3. Model of a deforming shear-band with heat and fluid fluxes

We consider a layer of saturated rock of thickness $h$ that is sheared in plane strain (Figure 3 ) in such a way that there is no extensional strain in the $x$-direction and displacements $u_{x}$, and $u_{z}$ of material points vary only with $z$ (and time $\mathrm{t}$ ). The stress state in the layer is a shear stress $\tau$, a normal stress, $\sigma$, in the $y$-direction. The only nonzero strains, a shear strain $\gamma$ and a normal strain $\varepsilon$, are related to the displacements by

$$
\gamma=\frac{\partial u_{x}}{\partial z} \quad \varepsilon=\frac{\partial u_{z}}{\partial z}
$$


Because the normal strains in the other directions are zero, the volumetric strain is equal to $\varepsilon$.

The equilibrium equations during quasi-static deformation reduce to:

$$
\frac{\partial \tau}{\partial z}=0 \quad \frac{\partial \sigma}{\partial z}=0
$$

so that the stresses must be spatially uniform and vary only with time during the deformation.

\section{Constitutive equations}

The rate thermo-poro elasto-plastic relationships are expressed as

$$
\dot{\gamma}=\frac{1}{G} \dot{\tau}+\dot{\gamma}^{p} ; \dot{\varepsilon}=\frac{1}{K}(\dot{\sigma}-b \dot{p})-\alpha_{s} \dot{T}+\dot{\varepsilon}^{p}
$$

where $G$ and $K$ are the elastic shear and bulk modulus respectively of the empty porous solid, $p$ is the pore pressure, $T$ is the temperature, $b$ is the Biot coefficient, $\alpha_{s}$ is the thermal dilation coefficient of the empty porous solid. The Biot coefficient is related to the bulk modulus of the solid matrix $K_{s}$ according to

$$
b=1-\frac{K}{K_{s}}
$$

The rate of plastic deformation is written as (Rice, 1975)

$$
\begin{aligned}
& \dot{\gamma}^{p}=\frac{1}{H}(\dot{\tau}-\mu(\dot{\sigma}-\dot{p})) \\
& \dot{\varepsilon}^{p}=\beta \dot{\gamma}^{p}
\end{aligned}
$$

where $H(\gamma)$ is the plastic hardening modulus, and is related to the tangent modulus $H_{\text {tan }}$ of the $\tau$ versus $\gamma$ curve by

$$
H_{\text {tan }}=\frac{H}{1+H / G}
$$

and is either positive or negative according to whether $\tau$ versus $\gamma$ curve is rising (hardening) or falling (softening), although the falling portion may never be observed as homogeneous deformation in a drained test. In equation [19], $\beta$ is the dilatancy coefficient. 


\section{Mass balance equation}

Conservation of fluid mass is expressed by

$$
\frac{\partial m_{f}}{\partial t}=-\frac{\partial q_{f}}{\partial z}
$$

where $m_{f}$ is the total fluid mass per unit volume of porous medium (in the reference state), and $q_{f}$ is the flux of fluid. The total fluid mass per unit volume of porous medium is written as $m_{f}=\rho_{f} n$, where $n$ is the pore volume fraction (Lagrangian porosity) and $\rho_{f}$ is the density of the saturating fluid. The left hand side of equation [21] is obtained by differentiating this product:

$$
\frac{\partial m_{f}}{\partial t}=n \frac{\partial \rho_{f}}{\partial t}+\rho_{f} \frac{\partial n}{\partial t}
$$

The derivatives of the right hand side of equation [22] are given by:

$$
\frac{\partial \rho_{f}}{\partial t}=\rho_{f} \beta_{f} \frac{\partial p}{\partial t}-\rho_{f} \lambda_{f} \frac{\partial T}{\partial t}
$$

and

$$
\frac{\partial n}{\partial t}=n \beta_{n} \frac{\partial p}{\partial t}+n \lambda_{n} \frac{\partial T}{\partial t}+\frac{\partial n^{p}}{\partial t}
$$

where $\beta_{f}=\frac{1}{\rho_{f}}\left(\frac{\partial \rho_{f}}{\partial P_{p}}\right)_{T}$ is the pore fluid compressibility, $\lambda_{f}=-\frac{1}{\rho_{f}}\left(\frac{\partial \rho_{f}}{T}\right)_{P_{p}}$ is the pore fluid thermal expansion coefficient of the pore fluid respectively, $\beta_{n}=\frac{1}{n}\left(\frac{\partial n}{\partial P_{p}}\right)_{T}$ is the pore volume compressibility and $\lambda_{n}=\frac{1}{n}\left(\frac{\partial n}{\partial T}\right)_{P_{p}}$ is the elastic thermal expansion coefficient of the pore volume, which is equal to the elastic thermal expansion coefficient of the solid fraction, $\lambda_{n}=\alpha_{s}$.

The expression of the pore volume compressibility is obtained from poroelasticity theory and is given by (Rice, 2006, Ghabezloo and Sulem, 2009) as:

$$
\beta_{n}=\frac{1}{n}\left(\frac{1}{K}-\frac{1+n}{K_{s}}\right)
$$


In equation [24], $\frac{\partial n^{p}}{\partial t}$ is the rate of plastic porosity change.

Using equations [2-4], the left hand side of equation [21] is thus evaluated as [see also Rice, 2006, Sulem et al., 2007]

$$
\frac{\partial m_{f}}{\partial t}=n \rho_{f}\left(\beta_{n}+\beta_{f}\right) \frac{\partial p}{\partial t}-\rho_{f} n\left(\lambda_{f}-\lambda_{n}\right) \frac{\partial T}{\partial t}+\rho_{f} \frac{\partial n^{p}}{\partial t}
$$

The flux term in equation [21] is evaluated assuming Darcy's law for fluid flow with viscosity $\eta_{f}$ through a material with permeability $k_{f}$

$$
q_{f}=-\frac{\rho_{f}}{\eta_{f}} k_{f} \frac{\partial p}{\partial z}
$$

Substituting [26] and [27] into [21] gives the equation which governs the pore fluid production and diffusion:

$$
\frac{\partial p}{\partial t}=c_{h y} \frac{\partial^{2} p}{\partial z^{2}}+\Lambda \frac{\partial T}{\partial t}-\frac{1}{\beta^{*}} \frac{\partial n^{p}}{\partial t}
$$

where:

$$
\Lambda=\frac{\lambda_{f}-\lambda_{n}}{\beta_{n}+\beta_{f}}
$$

is the thermo-elastic pressurization coefficient under undrained conditions (Rice, 2006). This coefficient is pressure and temperature dependent because the compressibility and the thermal expansion coefficients of the fluid vary with pressure and temperature, and also because the compressibility of the pore space of the rock can change with the effective stress (Ghabezloo \& Sulem, 2009). In equation [28], $\beta^{*}=n\left(\beta_{n}+\beta_{f}\right)$ is the storage capacity of the rock and $c_{h y}=k_{f} /\left(\beta \eta_{f}\right)$ is the hydraulic diffusivity. For incompressible solid phase and fluid, $\beta^{*}=1 / K$.

If we assume that the solid matrix is plastically incompressible,

$$
\frac{\partial n^{p}}{\partial t}=\frac{\partial \varepsilon^{p}}{\partial t}
$$




\section{Energy balance equation}

Let $E_{F}$ be the rate of frictional heat produced during shearing. The equation of conservation of energy is expressed as

$$
\rho C \frac{\partial T}{\partial t}=E_{F}-\frac{\partial q_{h}}{\partial z}
$$

where $\rho C$ is the specific heat per unit volume of the material in its reference state and $q_{h}$ is the heat flux. It is assumed here that all the plastic work is converted into heat and thus $E_{F}=\tau \dot{\gamma}^{p}$. The heat flux is related to the temperature gradient by Fourier's law:

$$
q_{h}=-k_{T} \frac{\partial T}{\partial z}
$$

where $k_{T}$ is the thermal conductivity of the saturated material. Substituting [32] into [31] gives the energy conservation equation:

$$
\frac{\partial T}{\partial t}=c_{t h} \frac{\partial^{2} T}{\partial z^{2}}+\frac{1}{\rho C} \tau \dot{\gamma}^{p}
$$

where $c_{t h}=k_{T} / \rho C$ is the thermal diffusivity.

\section{Undrained adiabatic limit}

We assume that the drainage and the heat flux are prohibited at the boundaries of the layer $\left(q_{f}=0\right.$ and $\left.q_{h}=0\right)$. We also assume that the normal stress $\sigma$ acting on the sheared layer is constant $(\dot{\sigma}=0)$. From equations [28], [30], [33], [19] we obtain the following relationship between the pore pressure rate and the shear stress rate:

$$
\dot{p}=\frac{\frac{\Lambda \tau}{\rho C}-\frac{\beta}{\beta^{*}}}{H-\mu\left(\frac{\Lambda \tau}{\rho C}-\frac{\beta}{\beta^{*}}\right)} \dot{\tau}
$$

Substituting equation [34] into equation [17] we get

$$
\dot{\gamma}=\left(\frac{1}{G}+\frac{1}{H+\mu \beta / \beta^{*}-\mu \Lambda \tau / \rho C}\right) \dot{\tau}
$$


or

$$
\dot{\tau}=\left(\frac{H+\mu \beta / \beta^{*}-\mu \Lambda \tau / \rho C}{1+\left(H+\mu \beta / \beta^{*}-\mu \Lambda \tau / \rho C\right) / G}\right) \dot{\gamma}
$$

If we neglect the thermal effect $(\Lambda=0)$ the expression for dilatant hardening effect as proposed by Rice (1975) is retrieved.

$$
\dot{\tau}=\left(\frac{H+\mu \beta / \beta^{*}}{1+\left(H+\mu \beta / \beta^{*}\right) / G}\right) \dot{\gamma}
$$

Equation [36] has to be compared with the drained case for which

$$
\dot{\tau}=\left(\frac{H}{1+H / G}\right) \dot{\gamma}
$$

For dilatant material $(\beta>0)$, we identify in equation [36] a hardening effect due to dilatancy with the term $\mu \beta / \beta^{*}$ and a thermal softening effect with the term $-\mu \Lambda \tau / \rho C$. When thermal softening prevails against strain hardening, plastic localization in the form of adiabatic shear banding can occur.

\section{Instability of the undrained adiabatic limit}

We consider again the shearing of the layer while drainage and heat fluxes are prevented at its boundaries. The pertinent variables are written in the form:

$$
\gamma=\gamma_{0}+\tilde{\gamma} ; \varepsilon=\varepsilon_{0}+\tilde{\varepsilon} ; \sigma=\sigma_{0}+\tilde{\sigma} ; \tau=\tau_{0}+\tilde{\tau} ; p=p_{0}+\tilde{p} ; T=T_{0}+\tilde{T}
$$

where the quantities $\gamma_{0}, \tau_{0}$, etc., represent the solution of the last section for homogeneous deformation and where $\tilde{\gamma}, \tilde{\tau}$ etc., are perturbation quantities. Specifically, the layer is sheared by application of a monotonically increasing shear stress $\tau_{0}=\tau_{0}(t)$ to its boundary while the normal stress is held constant at $\sigma_{0}$. From the equilibrium equation [16], the stress field is uniform within the layer, and thus $\tilde{\sigma}=0$ and $\tilde{\tau}=0$.This problem is similar to the one addressed by Rice (1975) with the introduction here of the thermal effect. From the conservation equations [28] and [33], and by keeping only the first order terms, we get the same type of rate equations for the perturbation terms: 


$$
\begin{aligned}
& \frac{\partial \tilde{p}}{\partial t}=c_{h y} \frac{\partial^{2} \tilde{p}}{\partial z^{2}}+\Lambda \frac{\partial \tilde{T}}{\partial t}-\frac{1}{\beta^{*}} \frac{\partial \tilde{\varepsilon}^{p}}{\partial t} \\
& \frac{\partial \tilde{T}}{\partial t}=c_{t h} \frac{\partial^{2} \tilde{T}}{\partial z^{2}}+\frac{1}{\rho C} \tau_{0} \frac{\partial \tilde{\gamma}^{p}}{\partial t}
\end{aligned}
$$

with from the constitutive equation [19]

$$
\frac{\partial \tilde{\gamma}^{p}}{\partial t}=\frac{\mu}{H} \frac{\partial \tilde{p}}{\partial t} ; \frac{\partial \tilde{\varepsilon}^{p}}{\partial t}=\beta \frac{\partial \tilde{\gamma}^{p}}{\partial t}=\beta \frac{\mu}{H} \frac{\partial \tilde{p}}{\partial t}
$$

The spatial dependence of the perturbations is decomposed into Fourier modes with wavelenth $\lambda$. Perturbations of the form

$$
\tilde{p}=P(0) e^{s t} \cos \left(\frac{2 \pi z}{\lambda}\right) ; \tilde{T}=T(0) e^{s t} \cos \left(\frac{2 \pi z}{\lambda}\right)
$$

with $\lambda=h / n$ and $n$ equal to an integer satisfy the zero heat and fluid flux boundary conditions at $y=0, h$. In equation [42], $s$ is the growth coefficient in time of the perturbation. By substituting the perturbations field (eq. [42]) into equation [40], we obtain a homogeneous algebraic system for the coefficients $P(0)$ and $T(0)$. Thus a nonzero solution is possible only if the determinant of the coefficients vanishes:

$$
\operatorname{det}\left(\begin{array}{cc}
s\left(1+\frac{\beta \mu}{\beta^{*} H}\right)+c_{h y}\left(\frac{2 \pi}{\lambda}\right)^{2} & -\Lambda s \\
\frac{\mu}{\rho C H} \tau_{0} s & -\left(c_{t h}\left(\frac{2 \pi}{\lambda}\right)^{2}+s\right)
\end{array}\right)=0
$$

The above condition yields a quadratic equation for the growth coefficient $s$ :

$$
\left(\frac{\Lambda \mu}{\rho C} \tau_{0}-\frac{\beta \mu}{\beta^{*}}-H\right) \lambda^{4} s^{2}-\left(\left(c_{t h}+c_{h y}\right) H+\frac{\beta \mu}{\beta^{*}}\right) \lambda^{2} s-c_{t h} c_{h y} H=0
$$

If a solution of [44] has a positive real part, then the corresponding perturbation grows exponentially in time.

If we do not consider the thermal effect, i.e. $\Lambda=0$, the condition for stability is simply $H>0$. Thus, the result of Rice (1975) is retrieved that undrained shearing is stable only in those circumstances for which the underlying drained deformation would be stable. This problem has also been examined by Vardoulakis (1996a,b) who proposed a regularization of the mathematically ill-posed problem in the softening regime by resorting to a second grade extension of plasticity theory. 
If we take now into account the thermal effect $(\Lambda>0)$, we observe that if $H>0$, the stability condition is

$$
\frac{\Lambda \mu}{\rho C} \tau_{0}<\frac{\beta \mu}{\beta^{*}}+H
$$

Consequently, the condition of stability of undrained adiabatic shearing is

$$
H>0 \text { and } \frac{\Lambda \mu}{\rho C} \tau_{0}<\frac{\beta \mu}{\beta^{*}}+H
$$

and the system is always unstable in the softening regime.

Obviously, this condition is more restrictive than the one for undrained shearing. This result demonstrate the destabilizing effect of thermal fluid pressurization: undrained adiabatic shearing of a material with positive strain hardening is stable only when the thermal pressurization is not too high.

\section{Conclusion}

Bifurcation analysis and localization theory constitute the basis of contemporary continuum theory of failure as a natural extension of classical theory of strength of materials. Major advances have been made possible thanks to the pioneering work of Professor Ioannis Vardoulakis during the three last decades.

\section{References}

Arthur, J.R.F., Dunstan, T., Al-Ani, Q.A.J., and Assadi, A.): Plastic deformation and failure in granular media, Géotechnique, 27, 1977, 53-74.

Bésuelle, P., Desrues, J., and Raynaud, S. Experimental characterisation of the localizationphenomenon inside aVosges sandstone in a triaxial cell. Int. J. Rock Mech. Min. Sci. 37, 8, 2000, 1223-1237.

Bésuelle, P., Rudnicki, J. Localization : Shear Bands and Compaction bands. In Mechanics of Fluid-Saturated Rocks. (Guéguen Y. and Boutéca M. edts), Academic Press, 2004, 219 321.

Chambon, R., Caillerie, D., Viggiani, G. Loss of uniqueness and bifurcation vs instability: some remarks. Revue Française de Génie Civil, Vol. 8, 5-6, 2004, 517-553.

Chau, K.T., Rudnicki, J.W. Bifurcation of compressible pressure sensitive materials in plane strain tension and compression. J. Mech. Phys. Solids, 38(6), 1990, 875-898.

Chester F.M. and Chester J.S., Ultracataclasite structure and friction processes of the Punchbowl fault, San Andreas system, California, Tectonophysics, 295, 1998, 199-221. 
Darve, F. Les lois incrementales non-linéaires, Manuel de rhéologie des géomatériaux, F. Darve, ed., Presse des Ponts et Chaussées, Paris, 1985.

Desrues, J. and Chambon, R. Shear band analysis for granular materials: The question of incremental non-linearity. Ingenieur Archiv, 59, 1989, 187-196.

Desrues, J., Chambon, R. Shear bands analysis and shear moduli calibration. Int. J. Solids Struct. 39: 2002, 3757-3776.

Desrues, J., Viggiani G., Strain localization in sand: an overview of the experimental results obtained in Grenoble stereophotogrammetry. Int. J. Num. Anal. Meth. Geomech., 28, 2004, 279-321.

El Bied, A., Sulem J. And Martineau F., Microstructure of shear zones in Fontainebleau sandstone. Int. J. Rock Mech. Min. Sci., 39, 7, 2002, 917-932.

Ghabezloo S., and Sulem J. 2009, Stress dependent thermal pressurization of a fluid-saturated rock. Rock Mechanics and Rock Engineering, 42, 1-24.

Germain, P. La méthode des puissances virtuelle en mécanique des milieux continus: Première partie: théorie du second gradient. Journal de Mécanique, 12, 1973a, 235-274.

Germain, P. The method of virtual power in continuum mechanics. Part 2: microstructure. SIAM J. Appl. Math. 25, 1973b, 556-575.

Hadamard, J. Leçons sur la propagation des ondes et les équations de l'hydrodynamique, Paris, 1903.

Hill, R., Acceleration waves in solids. J. Mech. Phys. Solids, 10, 1962, 1-16.

Issen, K.A., Rudnicki, J.W. Conditions for compaction bands in porous rock. J. Geophys. Res. 105, B9, 2000, 21529-21536.

Lyapunov, A.M. The general problem of stability of motion, 1892, English translation, Taylor $\&$ Francis.

Mandel, J. Propagation des surfaces de discontinuite dans un milieu élasto-plastique. In: Stress Waves in Anelastic Solids, Springer, 1964, 331-341.

Mandel, J. Conditions de stabilité et postulat de Drucker, Rheology and Soil Mechanics, Springer, 1966, 58-68.

Matsushima, T., Chambon, R., Caillerie, D. Large strain finite element analysis of a local second gradient model: application to localization. Int. J. Numer. Methods Eng. 54, 2002, 499-521.

Mindlin, R. Micro-structure in linear elasticity. Arch. Ration. Mech. An. 16, 1964, 51-78.

Mühlhaus H.-B. \& Vardoulakis, I. The thickness of shear bands in granular materials. Géotechnique, 37, 1987, 271-283.

Nova R. Controllability of geotechnical testing. Revue Française de Génie Civil, Vol. 8, 5-6, 2004, 613-634.

Nicot, F. and Darve, F. Diffuse and localized failure modes: Two competing mechanisms. Int. J. Num. Anal. Meth. Geomech., doi: 101002/nag912, 2010. 
Papamichos E., Vardoulakis I., Shear band formation in sand according to non-coaxial plasticity model, Géotechnique, 45, 4, 1995, 649-661.

Papanastasiou, P., Vardoulakis, I. Numerical analysis of progressive localization with application to borehole stability. Int. J. Num. Anal. Meth. Geomech., 13, 1992, 183-198.

Perrin, G., Leblond, J.B. Rudnicki and Rice's analysis of strain localization revisited. J. Appl. Mech. 60(4), 1993, 842-846.

Rice, J.R. On the stability of dilatant hardening for saturated rock masses. J. Geophys. Res. $80,11,1975,1531-1536$.

Rice, J.R. The Localization of Plastic Deformation, in: Theoretical and Applied Mechanics Proceedings of the 14th International Congress on Theoretical and Applied Mechanics, Delft, 1976, ed. W.T. Koiter, Vol. 1, North-Holland Publishing Co., 1976, 207-220

Rice, J.R. Heating and weakening of faults during earthquake slip, Journal of Geophysical Research, 111, 2006, B05311.

Rudnicki, J.W., Olsson,W.A. Reexamination of fault angles predicted by shear localization theory. Int. J. Rock Mech. Min. Sci., 35, 1998: 512-513.

Rudnicki J.W., Rice, J.R. Conditions for the Localization of Deformation in PressureSensitive Dilatant Materials, J. Mech. Phys. Solids, 23, 1975, 371-394

Sieffert, Y., Al Holo, S., Chambon, R. Loss of uniqueness of numerical solutions of the borehole problem modelled with enhanced media, Int. J. Solids and Struct. 46, 2009, 3173-3197

Simo, J.C., Oliver, J., Armero, F., An analysis of strong discontinuities induced by strainsoftening in rate-independent inelastic solids, Computational Mechanics, 12, 1993, 277 296.

Sulem, J. \& Vardoulakis, I. Analysis of the triaxial test on rocks specimens. A theoretical model for shape and size effect. Acta Mechanica, 83, 1990, 195-212.

Sulem J., Vardoulakis I., Papamichos E., Oulahna A.; Tronvoll, J. Elasto-plastic modelling of Red Wildmoor sandstone, Mechanics of Cohesive-Frictional Materials, 4, 1999, 215-245.

Sulem, J., I. Vardoulakis, H. Ouffroukh, M. Boulon, and J. Hans, "Experimental characterization of the thermo-poro-mechanical properties of the Aegion fault gouge", $C$. R. Geoscences, 336(4- 5), 2004, 455- 466.

Sulem, J., Ouffroukh, H. Shear banding in drained and undrained triaxial tests on a saturated sandstone: Porosity and permeability evolution, Int. J. Rock Mech. Min. Sci., 43, 2, 2006, 292-310.

Sulem, J., Lazar, P. and Vardoulakis, I. Thermo-Poro-Mechanical Properties of Clayey Gouge and Application to Rapid Fault Shearing, Int. J. Num. Anal. Meth. Geomechanics, 31, 2007, 523-540.

Sulem, J. and Famin, V. "Thermal decomposition of carbonates in fault zones: slipweakening and temperature-limiting effects", J. Geophys. Res., 114, B03309, 2009, doi:10.1029/2008JB006004.

Thomas, T.Y Plastic flow and fracture in solids, Academic Press, 1961. 
Vardoulakis, I., Equilibrium theory of shear band in plastic bodies. Mech. Res. Comm., 3, 1976, 209-214.

Vardoulakis, I. Shear band inclination and shear modulus of sand in biaxial tests. Int. J. Num. Anal. Meth. Geomech., 4, 1980, 3-119.

Vardoulakis, I. Deformation of water-saturated sand: I. uniform undrained deformation and shear banding. Géotechnique, 46, 3, 1996a, 441-456.

Vardoulakis, I. Deformation of water-saturated sand: II. effect of pore water flow and shear banding. Géotechnique, 46, 3, 1996a, 457-472.

Vardoulakis, I., Sulem, J., Bifurcation Analysis in Geomechanics, Chapman \& Hall, 1995.

Vermeer, P.A. and Schotman, G.J. An extension to the deformation theory of plasticity. Proc. 2nd Int. Symp. Num. Models Geomechan., Jackson and Son, England, 1986, 33-41.

Wibberley, C., Shimamoto, T. Internal structure and permeability of major strike-slip fault zones: the Median Tectonic Line in Mie Prefecture, Southwest Japan, J. Structural Geology, 25, 2003, 59-78.

Zervos A., Papanastasiou P., Vardoulakis I. Modelling of localization and scale effect in thick-walled cylinders with gradient elastoplasticity. Int. J. Solids Struct., 38, 2001, 5081-5095. 